\title{
Up-regulation of microRNA let-7c by quercetin inhibits pancreatic cancer progression by activation of Numbl
}

\author{
Clifford C. Nwaeburu ${ }^{1}$, Natalie Bauer ${ }^{1}$, Zhefu Zhao ${ }^{1}$, Alia Abukiwan ${ }^{1}$, Jury Gladkich ${ }^{1}$, \\ Axel Benner ${ }^{2}$, Ingrid Herr ${ }^{1}$ \\ ${ }^{1}$ Team Molecular OncoSurgery, Section Surgical Research, Department of General, Visceral and Transplantation Surgery, \\ University of Heidelberg, Heidelberg, Germany \\ ${ }^{2}$ Department of Biostatistics, German Cancer Research Center (DKFZ), Heidelberg, Germany \\ Correspondence to: Ingrid Herr, email: i.herr@uni-heidelberg.de \\ Keywords: cancer, microRNA, phytochemicals, stem cell signaling \\ Received: May 10, $2016 \quad$ Accepted: July 19, $2016 \quad$ Published: August 08, 2016
}

\section{ABSTRACT}

Pancreatic Ductal Adenocarcinoma (PDA) is a highly malignant tumor with poor prognosis. MicroRNAs (miRs) may offer novel therapeutic approaches to treatment. The polyphenol quercetin, present in many fruits and vegetables, possesses anticarcinogenic properties. To unravel the effect of quercetin to miR signaling we performed miR profiling in PDA cells before and after quercetin treatment, followed by biostatistical analysis. miR let-7c was among the top up-regulated candidates after quercetin treatment, as measured by qRT-PCR and confirmed in two established and one primary PDA cell lines. By computational analysis we identified the Notchinhibitor Numbl as let-7c target gene. This was strengthened by luciferase assays, where lipofected let-7c mimics induced a Numbl 3-UTR wild type construct, but not the mutated counterpart. Let-7c induced Numbl mRNA and protein expression but inhibited Notch just like quercetin. It also inhibited colony formation, wound healing, and protein expression of progression markers. In vivo xenotransplantation of PDA cells and subsequent intravenous injection of let-7c resulted in a significant decrease in tumor mass without obvious toxic effects in the fertilized chick egg model. The delivery rate of the miR mimics to the tumor mass was $\mathbf{8 0 \%}$, whereas minor amounts were present in host tissue. By immunohistochemistry we demonstrated that let-7c inhibited Notch and progression markers but up-regulated Numbl. These findings show that quercetin-induced let-7c decreases tumor growth by posttranscriptional activation of Numbl and indirect inhibition of Notch.

\section{INTRODUCTION}

Pancreatic ductal adenocarcinoma (PDA) is an extremely lethal disease that is mostly diagnosed in an advanced state for which there are little or no effective therapies [1]. It's 5-year survival rate is about 5\%, making it one the worst prognosis of any major malignancy [2]. Notwithstanding the extensive progress in understanding oncogenic-signaling networks, effective therapies remain scarce due to high drug resistance of PDA.

MicroRNAs (miRs) are important players in the chemoresistance of PDA [3, 4]. They are small noncoding RNAs that regulate gene expression by sequencespecific binding to target genes [5]. miRs mediate post- transcriptional down-regulation, translational repression and deadenylation-dependent decay of messages via partially complementary miR target sites in untranslated regions (UTRs) of mRNAs. They have also been found to post transcriptionally stimulate gene expression by direct and indirect mechanisms [6]. Aberrant miR expression and functions are linked to several types of tumors, immune diseases and developmental disorders and differentially expressed miRs may also offer novel therapeutic approaches for future, more successful treatment of PDA $[3,4]$.

The miR-let-7 (from "lethal") is one of the first identified miRs [7] and the expression levels of let-7 members are significantly low in human cancers. The 
major function of the let-7 family is to target genes, which promote the terminal differentiation in development, and to suppress tumor growth by targeting oncogenes such as Myc and Ras [6]. Let-7 miRs have also been identified to regulate Notch signaling pathways [8], known to regulate many cellular processes, including cell proliferation, apoptosis, migration, invasion, angiogenesis and normal development [9]. Notch signaling is initiated by the binding of the Notch trans-membrane receptors with their specific ligands between 2 adjoining cells [10]. An inhibitor of Notch expression is the Numb/Numblike (Numb), involved in cell fate determination and asymmetric cell division [11].

Recently, we demonstrated the up-regulation of let-7a by quercetin in PDA cells by qRT-PCR analysis [12]. Quercetin is a fruit-and vegetable-derived flavonoid, present in high concentration in e.g. berries, tea leaves, onions, broccoli and apples [13]. In addition, quercetin has been shown to decrease the level of Notch1 protein in a leukemia cell line by acting as a Wnt inhibitor [14]. It is still unknown if quercetin may also lead to Notch downregulation in PDA and if it is mediated by let-7 signaling.

In the present study, we demonstrate by miR profiling that members of the let-7 family are in the top upregulated miRs after quercetin treatment of PDA cells. With focus on let-7c, we identified its binding to the 3 'UTR of Numbl. Let-7c mimic or quercetin enhanced the expression of Numbl, but reduced the expression of Notch. This led to inhibition of clonogenicity, migration and expression of progression markers. The injection of a let$7 \mathrm{c} /$ lipofectamine mixture or of quercetin into the vessels of the chorioallantoismembrane (CAM) of fertilized chick eggs xenotransplanted with PDA cells reduced the tumor size and induced an expression pattern in tumor tissue similar to those observed in vitro. These results suggest a high dietary intake of quercetin or systemic let-7c liposomal application as supporting therapeutic treatment for patients with PDA. In addition, our data introduce the injection of a miR/liposome mixture into the CAM of chick eggs harboring xenograft tumors as a new model for easy-to-perform and cost-effective in vivo miR studies in cancer research.

\section{RESULTS}

\section{Quercetin upregulates let-7c}

To determine the impact of quercetin to $\mathrm{miR}$ regulation, we performed a miR microarray expression profiling of AsPC-1 cells before and after treatment with quercetin for $12 \mathrm{~h}$ (Supplementary Figure 1A-1D). By this way, 105 differentially and statistically significant expressed miRs were identified in quercetin-treated cells with an adjusted p-value less than 5\%. Among the top 24 differentially regulated candidates were several miRs, which were known to be deregulated in pancreatic cancer, among them the miR-29 and let-7 families (Figure 1A). To identify the most relevant miR candidates and their potential target genes, we performed in-silico analysis using the Ingenuity ${ }^{\circledR}$ Pathway Analysis (IPA), miRanda, TargetScan and PicTar miRNA databases. Since our interest was in tumor progression, we selected Notch and Numbl as target genes of interest. This search resulted in the selection of 11 miRs out of the 105 (Table 1). To check the accuracy of the microarray-based miRNA quantification, 6 out of the selected 11 miRs were confirmed by qRT-PCR. According to the microarray data, quercetin treatment led to the up-regulation of let-7c, miR-200a-3p and miR-200b-3p and the down-regulation of miR-103a-3p, miR-125b-5p and miR-1202 (Figure 1B). Based on these results and published data $[15,16]$, let-7c was selected as most relevant candidate miR. For confirmation purposes, the expression of let-7c was analyzed in the established PDA cell line PANC-1 and in the primary PDA cell line ASANPaCa before and after treatment with quercetin for $12 \mathrm{~h}$ and compared to AsPC-1 cells. The qRT-PCR results showed that the levels of let-7c in the untreated samples across all cell lines were low but significantly increased in the quercetin treated samples (Figure 1C), thus confirming our previous results. Sequel to this, we hypothesized that the expression of let-7c is low in pancreatic cancer. To authenticate our hypothesis, we evaluated the let-7c levels in normal pancreas fibroblasts (CRL-4023) and PDA cell lines (AsPC-1, ASANPaCa and PANC-1) using qRT-PCR. We observed a significantly higher level of let-7c expression in the normal pancreas fibroblast as compared to the PDA cell lines thus confirming our hypothesis (Figure 1D).

\section{Let-7c directly targets the Numbl 3'-UTR}

To validate Numbl as a target gene for let-7c, firstly, the putative binding site of let-7c in the Numbl 3'-UTR was identified using the TargetScan database and the resulting sequence homology at base pairs 626-632 are shown (Figure 2A, Supplementary Data 1). Then we mutated the miR seed-binding regions by triplet substitution and confirmed the successful mutation by DNA sequencing. AsPC-1 and PANC-1 cells were cotransfected with a reporter construct of the human wild type or mutant Numbl 3'-UTR or with control firefly plasmid together with either let-7c miR mimic or a control miR. Forty-eight hours later we performed a luciferase assay. Let-7c significantly suppressed luciferase expression with respect to control miRNA for the wild type, but not for the mutated Numbl 3'-UTR (Figure 2B). Hence, let-7c functionally interacts with the Numbl 3'UTR. These results show for the first time, that Numbl is a direct target gene for let-7c. 


\section{Let-7c inhibits Notch and activates Numbl}

Next we asked if the overexpression of let-7c might regulate the expression of Numbl. AsPC-1 cells were transfected with let-7c mimic or non-coding miR, which served as control together with untreated cells. In addition, we treated non-transfected cells with quercetin for $12 \mathrm{~h}$, followed by qRT-PCR, immunofluorescence and Western blot analysis of Numbl and Notch expression. On the mRNA level, the expression of
Numbl was more than $50 \%$ lower than Notch in control cells, but about $150 \%$ higher in let- $7 \mathrm{c}$-expressing or quercetin-treated cells (Figure 2C). Likewise, we saw low Numbl and high Notch expression in control cells, but the opposite in let-7c-transfected or quercetin-treated cells (Figure 2D, 2E). Thus, there are high endogenous levels of Notch, but low levels of Numbl in PDA cells; but upon let-7c transfection or quercetin the expression of Numbl increases and in turn inhibits Notch.
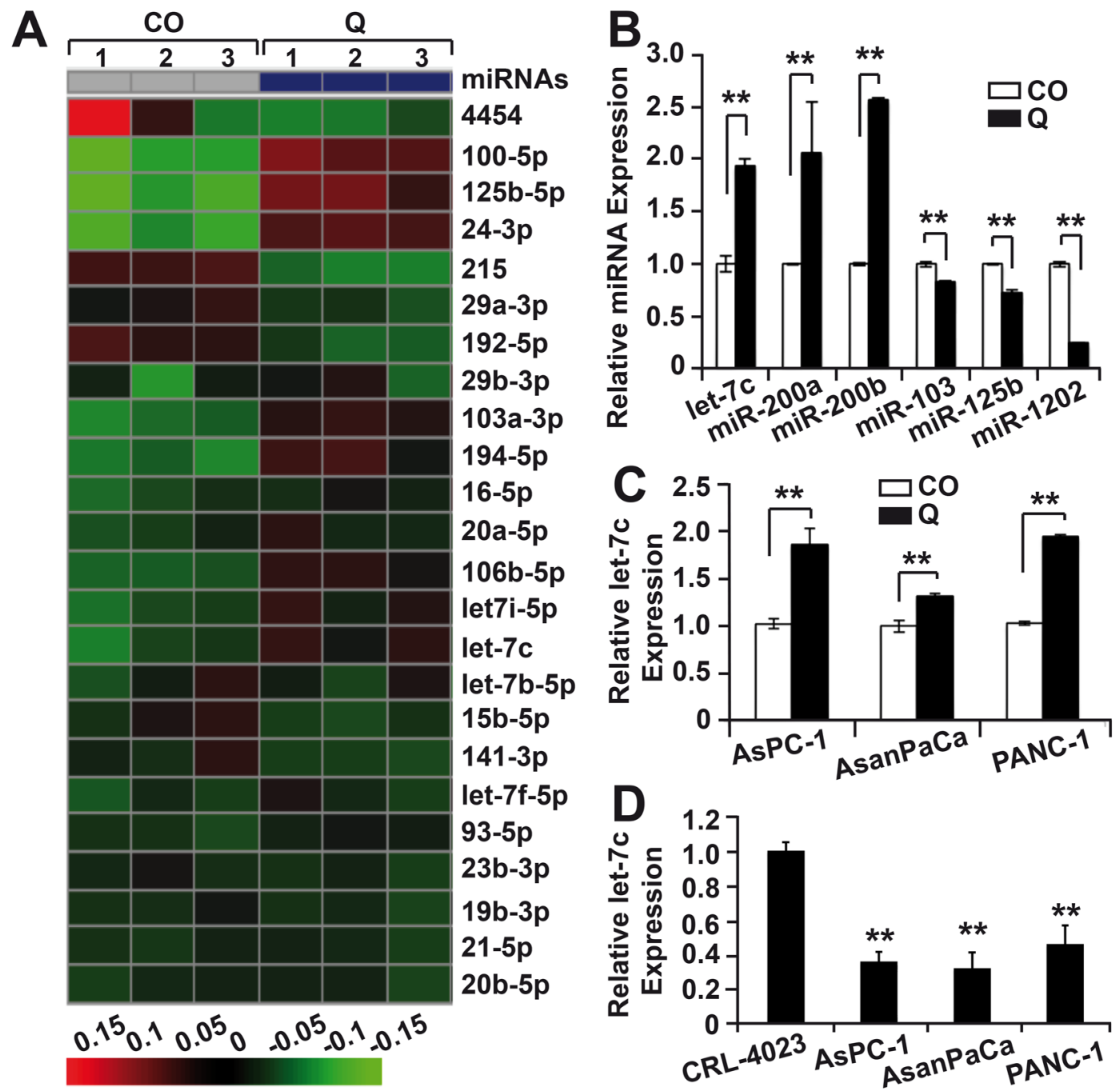

Figure 1: MiR let-7c is deregulated in pancreatic cancer. A. AsPC-1 cells were left untreated (CO) or were treated with quercetin $(\mathrm{Q}, 50 \mu \mathrm{M}) .12$ hours later, the RNA was harvested with a Qiagen miRNeasy mini isolation kit and analyzed by the use of the Agilent miRNA Microarray (Release 19.0). The heatmap presents the top 24 most significantly differentially up- and down-regulated miRs. The red colors mark high expression and the green colors low expression within a scale from 0.15 to -0.15 . B. The microarray data were validated with a qRT-PCR using 6 of the miRNA candidates. For that, AsPC-1 cells were treated again as described in point A, followed by isolation of miRs and qRT-analysis. The expression levels were normalized to RNU44 and the mean fold change of the untreated (CO) AsPC-1 cells were set to 1, whereas the expression level in the quercetin-treated cells was related to the control. C. After in silico analysis, the qRT-PCR was repeated as described above using the selected candidate let $7 \mathrm{c}$ on 3 different pancreatic cancer cell lines; AsPC-1, ASANPaCa and PANC-1. The experiments were repeated three times and the data are presented as mean $\pm \mathrm{SD}$. D. The difference in let-7c expressions levels between normal pancreatic duct fibroblast (CRL-4023) and pancreatic cancer cell lines was evaluated with qRT-PCR. The mean fold change of CRL-4023 was set to 1 . 
Table 1: Summary of in-silico analysis

\begin{tabular}{|c|c|c|c|}
\hline ID & Confidence & Gene & Pathway \\
\hline let-7c & High (predicted) & NUMBL & Notch Signaling \\
\hline $\operatorname{miR}-103 a-3 p$ & Moderate (predicted) & NUMB & $\begin{array}{c}\text { Clathrin-mediated } \\
\text { Endocytosis Signaling, } \\
\text { Notch Signaling }\end{array}$ \\
\hline miR-1202 & High (predicted) & NUMBL & Notch Signaling \\
\hline $\operatorname{miR}-125 b-5 p$ & High (predicted) & NUMBL & Notch Signaling \\
\hline miR-1268a & Moderate (predicted) & NUMBL & Notch Signaling \\
\hline miR-200a-3p & High (predicted) & NUMBL & Notch Signaling \\
\hline miR-6088 & Moderate (predicted) & NUMB & $\begin{array}{c}\text { Clathrin-mediated } \\
\text { Endocytosis Signaling, } \\
\text { Notch Signaling }\end{array}$ \\
\hline $\operatorname{miR}-200 b-3 p$ & High (predicted) & NUMB & $\begin{array}{l}\text { Clathrin-mediated } \\
\text { Endocytosis Signaling, } \\
\text { Notch Signaling }\end{array}$ \\
\hline $\operatorname{miR}-3665$ & Moderate (predicted) & NUMB & $\begin{array}{c}\text { Clathrin-mediated } \\
\text { Endocytosis Signaling, } \\
\text { Notch Signaling }\end{array}$ \\
\hline $\operatorname{miR}-4651$ & High (predicted) & NUMBL & Notch Signaling \\
\hline miR-96-5p & High (predicted) & NUMB & $\begin{array}{c}\text { Clathrin-mediated } \\
\text { Endocytosis Signaling, } \\
\text { Notch Signaling }\end{array}$ \\
\hline
\end{tabular}

\section{Let-7c inhibits progression features}

Next, we questioned if the activation of let-7c would have an effect on cancer progression features. To this end, AsPC-1, ASANPaCa and PANC-1 cells were transfected with let-7c mimic or a control miR or were treated with quercetin for $12 \mathrm{~h}$ or not, followed by colony formation assays, wound healing assays and Western blot analysis of progression markers. Compared to control cells, we observed significant inhibition of colony formation in let-7c-expressing or quercetin-treated cells (Figure 3A3C). More so, considerable inhibition of the wound healing capacity occurred (Figure 3D, 3E, Supplementry Figure $1 \mathrm{E}$ and $1 \mathrm{~F}$ ). Likewise, a down regulation of the cancer stem cells markers ALDH1, CD24 and CD44 was demonstrated by Western blot analysis (Figure 3F).

\section{Osteoblastic differentiation is induced by let-7c}

Since the Notch signaling pathway is vital in osteoblastogenesis and the expression of Numbl is known to promote differentiation, we questioned the role of let-7c in osteoblastic differentiation. Cells were left un-transfected, treated with quercetin for $96 \mathrm{~h}$ or were transfected with let-7c mimics or for $96 \mathrm{~h}$. Osteoblastic differentiation was detected by staining of cells with BCIP/
NBT to examine alkaline phosphatase (ALP) and calcium phosphate deposits. Quercetin- and let-7c-treated AsPC1 and ASANPaCa cells were positive for heterogeneous alkaline phosphatase activity indicating osteoblastic differentiation while the controls did not differentiate (Figure 4A). No significant osteoblastic differentiation result was obtained for PANC1 (result not shown).

\section{Let-7c decreases tumor growth in vivo}

Having established the functional effects of let-7c in vitro, we questioned the possibility of reproducing the effects in tumor xenotransplants on fertilized chick eggs. This in vivo system is naturally immunodeficient and therefore tolerates the transplantation of human tumor cells. For this purpose, we selected the primary human PDA cell line ASANPaCa, since it is more representative of the key functional constituent of the source tissue than the immortalized cells and hence, can be used to resolve accuracy of extrapolating human data from an animal model.

The chick embryo has an incubation period of 21 days, so we set up our experimental design as follows: clean the eggs with $70 \%$ ethanol and place them on the incubator on day 1; open the eggs and remove the unfertilized/spoilt eggs on day 4; transplant the tumor 
A

3'UTR Numb
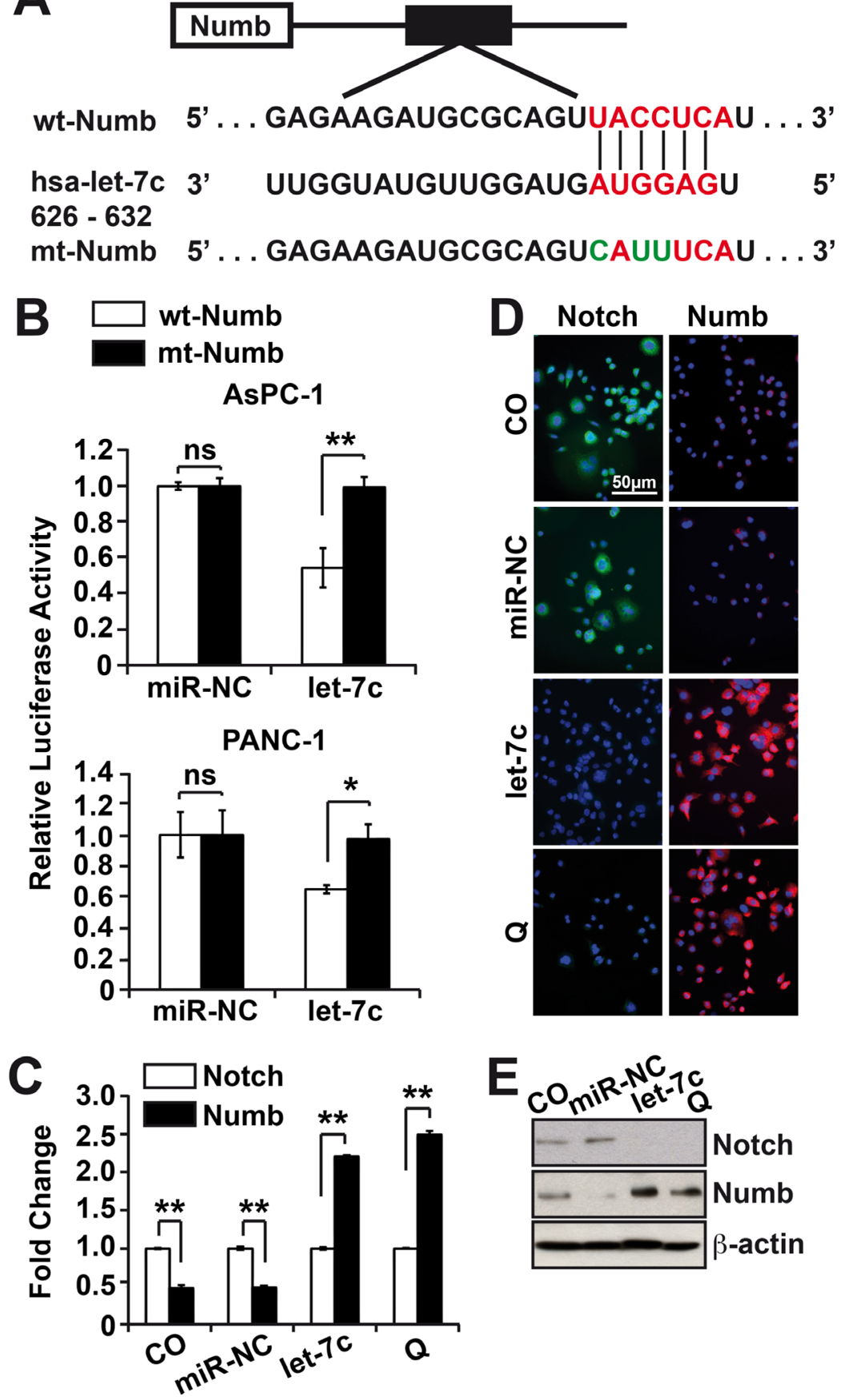

Figure 2: Numbl is a direct target of miR let-7c. A. Putative binding sites of let-7c in the wild type Numbl 3'-UTR were identified using the TargetScan database and the resulting sequence homology at position 626-632 of the Numbl 3'-UTR are shown. The seed-binding region (marked in red) was mutated by triplet substitution (shown in green) using the QuickChange Site-Directed Mutagenesis kit. B. The wild type and mutant Numbl 3'-UTRs were cloned into a pLightSwitch renilla plasmid or empty vector and lipofected into AsPC-1 or PANC-1 cells in presence or absence of $50 \mathrm{nM}$ let-7c mimic or a non-coding miR control (miR-NC). The co-transfection of a firefly luciferase $(0.25 \mathrm{ng} / \mu \mathrm{l})$ served as control for equal conditions. 48 hours after transfection, the expression of renilla and firefly luciferase was detected on a FLUOstar Omega microplate reader. Renilla luciferase activities were normalized to firefly luciferase activities. C. AsPC-1 cells were lipofected as described above or were treated with quercetin $(\mathrm{Q}, 50 \mu \mathrm{M})$ or not for $12 \mathrm{~h}$. Total RNAs were harvested $48 \mathrm{~h}$ after transfection and total proteins were harvested $72 \mathrm{~h}$ later. Notch 1 and Numbl mRNA levels were analyzed by qRT-PCR. GAPDH served as control for equal conditions. D. AsPC-1 cells were treated as described above and subsequently, immunofluorescence staining for Notch and Numbl was performed. E. Total proteins obtained as described in point $\mathrm{C}$ were analyzed by Western blot. $\beta$-actin served as control for equal conditions. All assays were performed in triplicates, repeated at least three times and the means $\pm \mathrm{SD}$ are shown. 
A AsPC-1
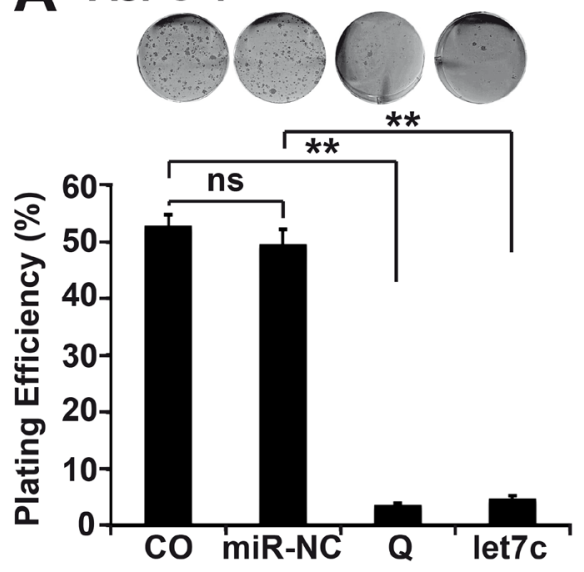

B AsanPaCa Cells
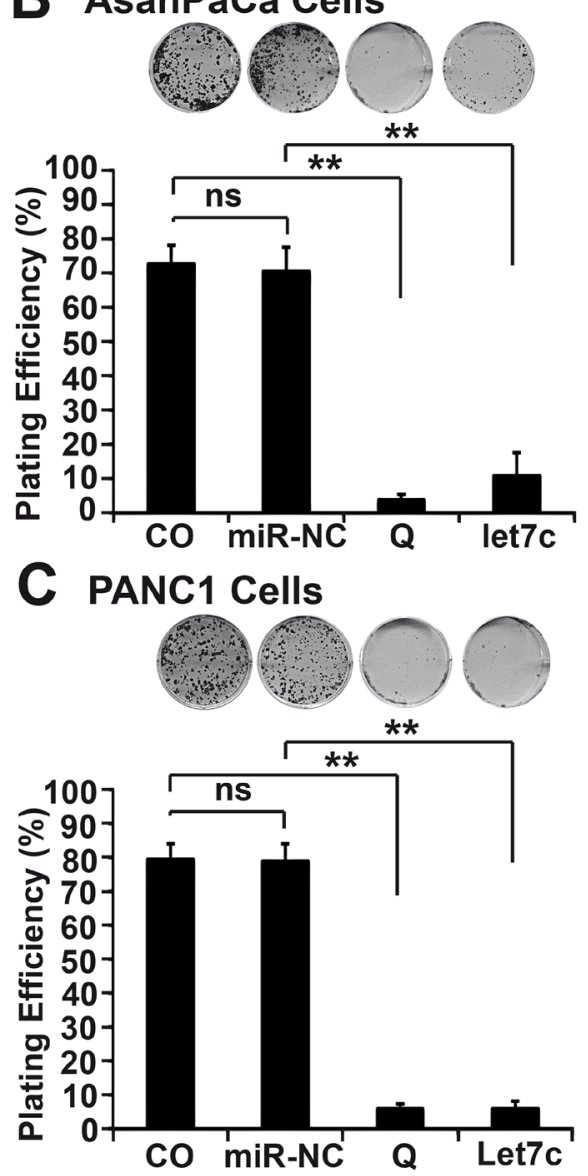

D
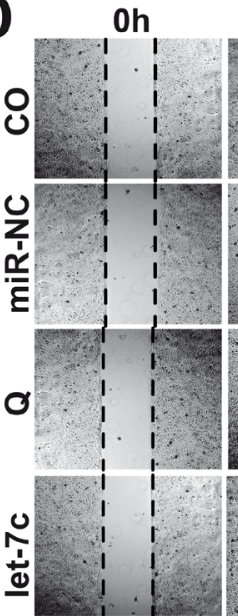

$24 h$

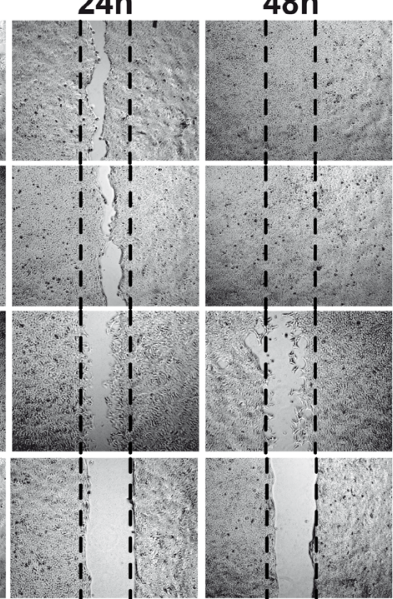

$\mathbf{E}$

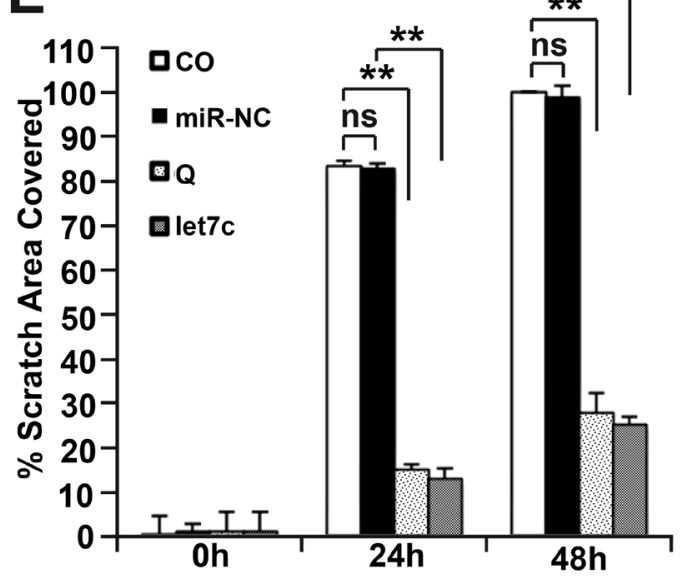

$\mathbf{F}$

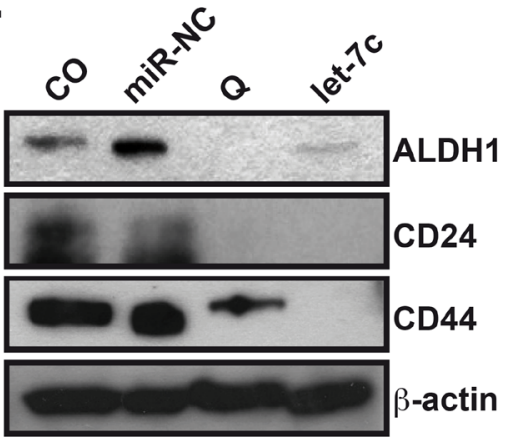

Figure 3: Let-7c inhibits cancer stem-like cells in vitro. A. AsPC-1 cells were harvested $24 \mathrm{~h}$ after lipofection with $50 \mathrm{nM}$ let-7c mimic or non-coding miR control (miR-NC) or were treated with quercetin (Q) or not (CO). Then the cells were resuspended in DMEM medium supplemented with $10 \%$ FCS and plated at a density of 500 cells/well in 6-well tissue culture plates. The cultures were maintained under standard culture conditions for fourteen days followed by evaluation of fixed and Coomassie-stained colonies consisting of at least 50 cells. The plating efficiency as a percent was calculated (number of colonies/number of seeded cells) $\times 100$. B. The above was repeated for ASANPaCa cells and C. for PANC-1 cells. D. AsPC-1 cells were seeded at high density in Ibidi culture-Insert 24 plates. Twenty-four hours after seeding, when the cells attached and reached about $90 \%$ confluency, the inserts were removed thereby leaving a nice $500 \mu \mathrm{m}$ thick wall (wound) and the cells were lipofected with $50 \mathrm{nM}$ let-7c mimics or non-coding miR control (miR-NC) or were treated with $(\mathrm{Q}, 50 \mu \mathrm{M})$ or were left untreated (CO). Images of the cell-free gap were obtained immediately as $0 \mathrm{~h}$ and subsequently $24 \mathrm{~h}$ and $48 \mathrm{~h}$ after treatment. E. Images obtained from above were analyzed using Ibidi's WimScratch software and the percentage of the wounded area covered by the cells from the different treatment groups evaluated. F. Total proteins were obtained from AsPC-1 cells after treatment as described in A. Western blot analyses was performed for the cancer stem cell markers ALDH1, CD24 and CD44. $\beta$-actin served as controls for equal conditions. All assays were performed in triplicates, repeated at least three times and the means \pm SD are shown. 
cells on day 9; inject miRs on day 15 in CAM vessels leading to the tumor, or treat with quercetin by applying it on an autoclaved filter paper on the CAM; resect the xenografts on day 18 and subsequently analyze the tumor sizes (Figure 5A). The tumors obtained from the control and groups were significantly bigger than those from the let-7c- or quercetin-treated groups (Figure $5 \mathrm{~B}$ and $5 \mathrm{C}$ ), which did not differ significantly. This suggests that let$7 \mathrm{c}$ or quercetin decreases tumor growth in vivo. Next we checked side effects of the treatment by physically examining the chicks from each group for teratogenicity and we discovered none (data not shown). Then we weighed all the chicks from each group and their mean weights showed no significant difference (Figure 5D). Furthermore, we performed a Hematoxylin and Eosin (HE) staining on the liver tissues obtained from the different groups and no morphological differences were found (Figure 5E). To verify the amount of let-7c that was actually delivered to the tumor and other tissues, we performed a qRT-PCR using the CAM, liver, tumor and lungs tissues. We observed that most of the let-7c (80.3\%) was delivered to the tumor, followed by the liver $(17.6 \%)$ and then the CAM (2.1\%) (Figure 5F and 5G). These data suggest that the treatment of xenografted fertilized chick eggs is an efficient and effective method for delivery of miR and therapeutic treatment, which reflects the results obtained in vitro.

\section{Let-7c inhibits marker expression for proliferation and progression in tumors}

To highlight our in vivo results, we obtained frozen tissue sections of the tumors from each group and performed immunofluorescence, immunohistochemical and western blot analyses. In correlation to our in vitro results, the immunofluorescence staining of Notch showed high expression levels in the control groups that decreased in the treatment groups. The opposite was found for Numbl (Figure 6A). Next, we evaluated the expression of the proliferation marker Ki67 and of the cleaved fragment of activated caspase-3, which indicates apoptosis. Whereas Ki67 expression and thus proliferation was high in the control groups, it decreased after treatment (Figure 6B and 6C). Vice versa, caspase-3 staining and thus apoptosis was low in the control groups, but increased about 50\% after treatment (Figure 6B and 6C). These data suggest that let-7c or quercetin inhibits proliferation and activates apoptosis. We evaluated the in vivo effect of let-7c on cancer stem cell surface markers by staining for CD24 and CD44. The double immunofluorescence staining showed a down regulation of these markers by let-7c or quercetin (Figure 6D). Finally, by western blot analyses we replicated the results obtained in vivo and in vitro (Figure 6E).

\section{DISCUSSION}

In the present study, we show that quercetin upregulates microRNA let-7c, which decreases tumor growth by posttranscriptional activation of Numbl and subsequent inhibition of Notch. Let-7c is observed to function like quercetin in the induction of Numbl expression, inhibitions of Notch, colony formation, wound healing and protein expression of progression markers. By the use of the chick egg xenograft model, we demonstrate for the first time, a cost-effective, quick and efficient in vivo miR delivery method, which reflects the in vitro results.

Quercetin enriched nutrition is without doubt beneficial to mankind. It has even been suggested to reduce the risk of pancreatic cancer [17]. We used 50 $\mu \mathrm{M}$ concentrations of quercetin since concentrations more than $200 \mu \mathrm{M}$ are impractical in the clinical settings, where peak plasma concentrations of $108.7 \pm 41.67 \mu \mathrm{M}$
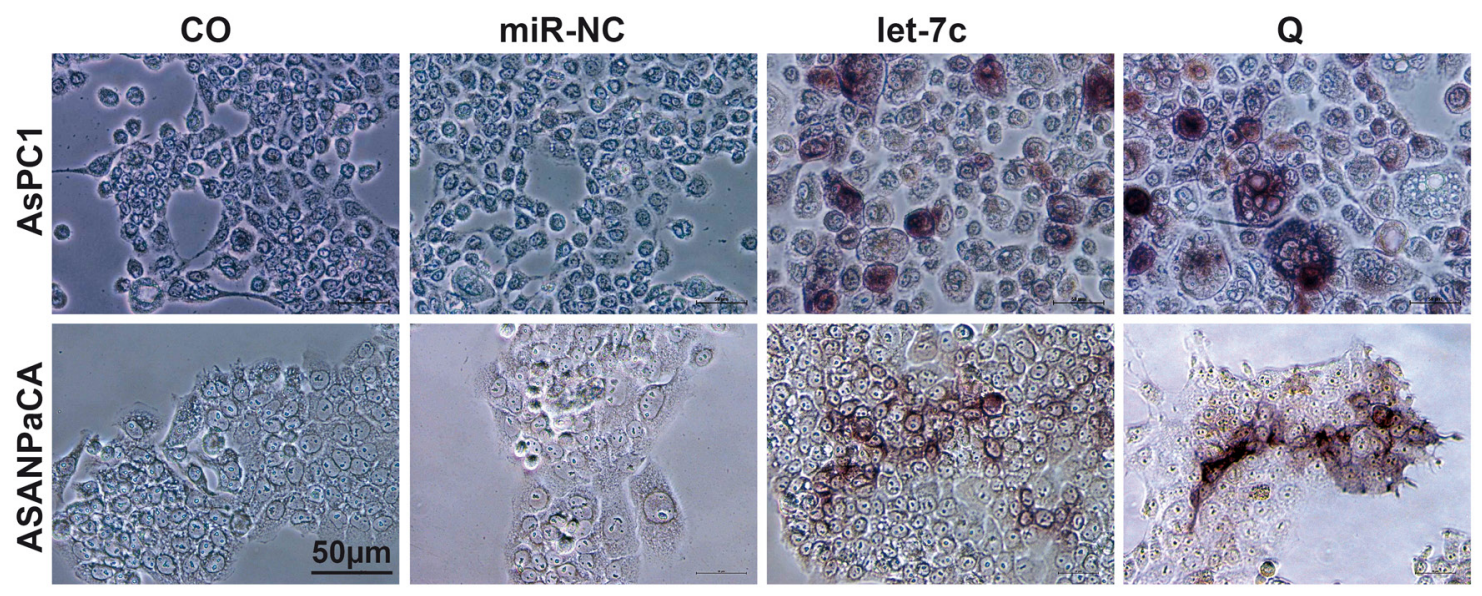

Figure 4: Let-7c induces osteoblastic differentiation. AsPC-1 and ASANPaCa cells were lipofected with $50 \mathrm{nM}$ let-7c or noncoding miR control (miR-NC) mimics for $72 \mathrm{~h}$ or treated with quercetin $50 \mu \mathrm{M}$ for $12 \mathrm{~h}(\mathrm{Q})$ or were left untreated (CO). Osteoblastic differentiation was detected using Sigma Fast BCIP/NBT substrate. The positive cells appear dark brown/purple. The bar indicates $50 \mu \mathrm{m}$. 


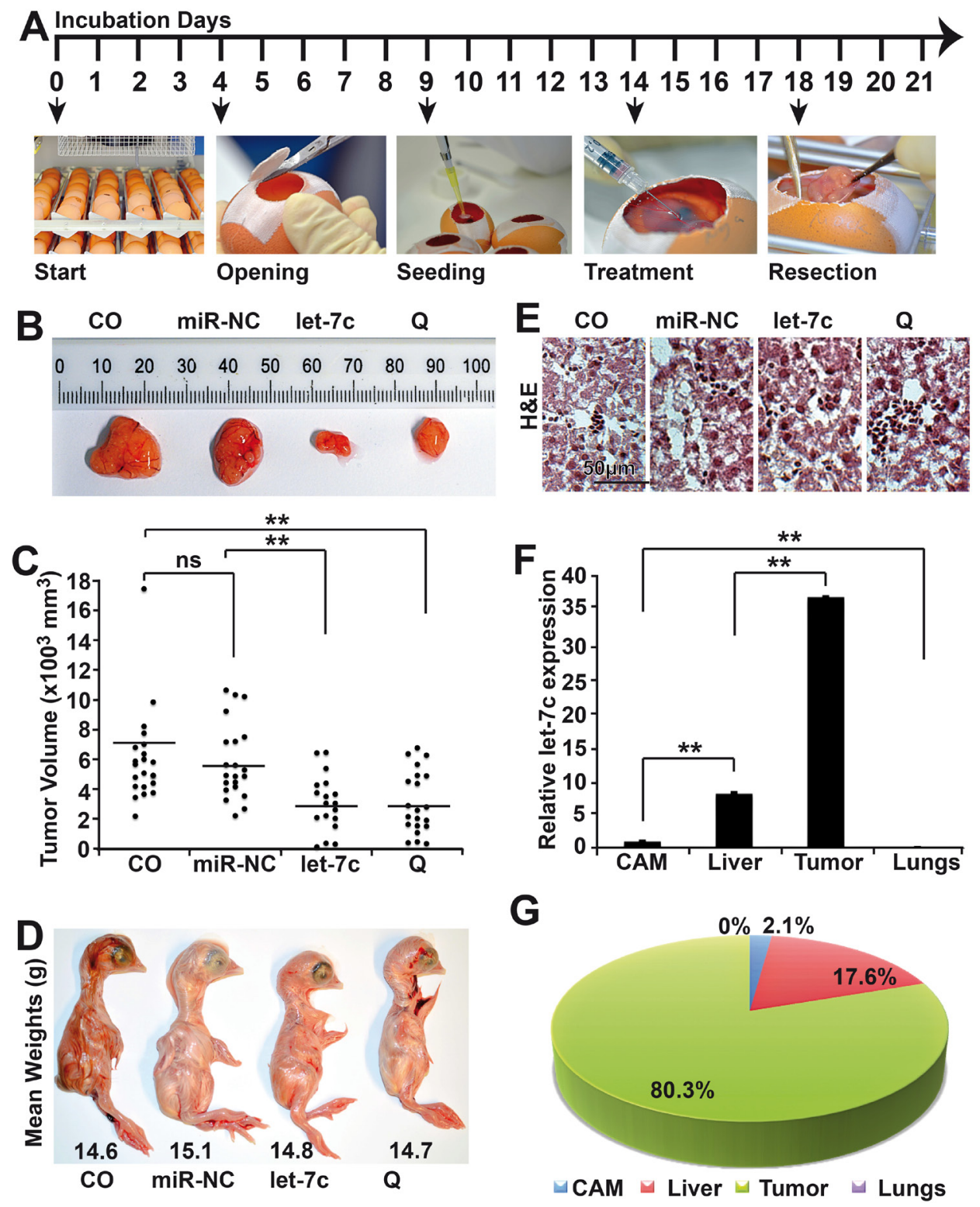

Figure 5: Let-7c reduces the volume of xenograft tumors. A. Experimental scheme for the in vivo miR delivery. Immediately after delivery, fertilized chick eggs were properly cleaned with $70 \%$ ethanol and placed in the incubator at $37^{\circ} \mathrm{C}$ with $60 \%$ humidity and this is the Egg Development Day 0 (EDD 0). On the EDD 4, the eggs were taken out of the incubator, cleaned again with $70 \%$ ethanol and a piece of leukosilk placed on the eggshell. About $3 \mathrm{ml}$ albumin was removed using a sterile syringe and needle. The egg shell was cut open and the embryo evaluated for viability. The albumin was re-injected and the opening closed with leukosilk. The egg was properly labeled and placed back to the incubator. On EDD 9, the tumor cells $\left(5 \times 10^{5}\right.$ cells/egg in $25 \mu 1$ medium) were mixed 1:1 with matrigel and placed on ice. A sterile coverslip with a hole at the center was placed on the CAM of the egg and gently scratched. Fifty-microliter of the tumor cell/matrigel mixture was seeded per egg onto the coverslip. On the EDD 14, $50 \mathrm{nM}$ of let-7c mimic or non-coding miR control reverse transfection mixture were mixed with chicken saline in the ratio of 1:1 to ensure the chick embryo's system do not reject it and delivered intravenously into any of the blood vessels leading directly to the tumor. Quercetin treatment was performed by its application on an autoclaved filter paper on the CAM. On the EDD 18, the tumors were resected from the eggs using sterile forceps and scissors. The embryo was euthanized by the injection of $0.05 \mathrm{ml}$ Narcoren per egg ( $16 \mathrm{~g}$ Pentobarbital/100 $\mathrm{ml}$ ). B. At day 18 of embryonic development, the xenografts were resected from eggs in which the embryos were alive at least until day 17 and the size of each tumor was determined using calipers. Representative xenografts from each treatment group are shown. C. The tumor volumes are presented as block dots and the volume was set to zero were no tumor grew. The bar indicates the median of the tumor volume in each experimental group. D. Representative images from the chicks from the different experimental groups. E. H\&E staining of the frozen liver section $(\times 100$ magnification). F. Total RNA was extracted from the lungs, liver, CAM and tumors from the let-7c-treated groups. The amount of let- $7 \mathrm{c}$ in the various tissues was evaluated by qRT-PCR. The expression levels were normalized to RNU44 and the expression levels in the different tissues were related to the CAM. G. MicroRNA let-7c expression observed in the different tissues was related to percentages. All assays were performed in triplicates, repeated at least three times and the means \pm S.D. are shown. $* \mathrm{P}<0.05, * * \mathrm{P}<0.01$. 


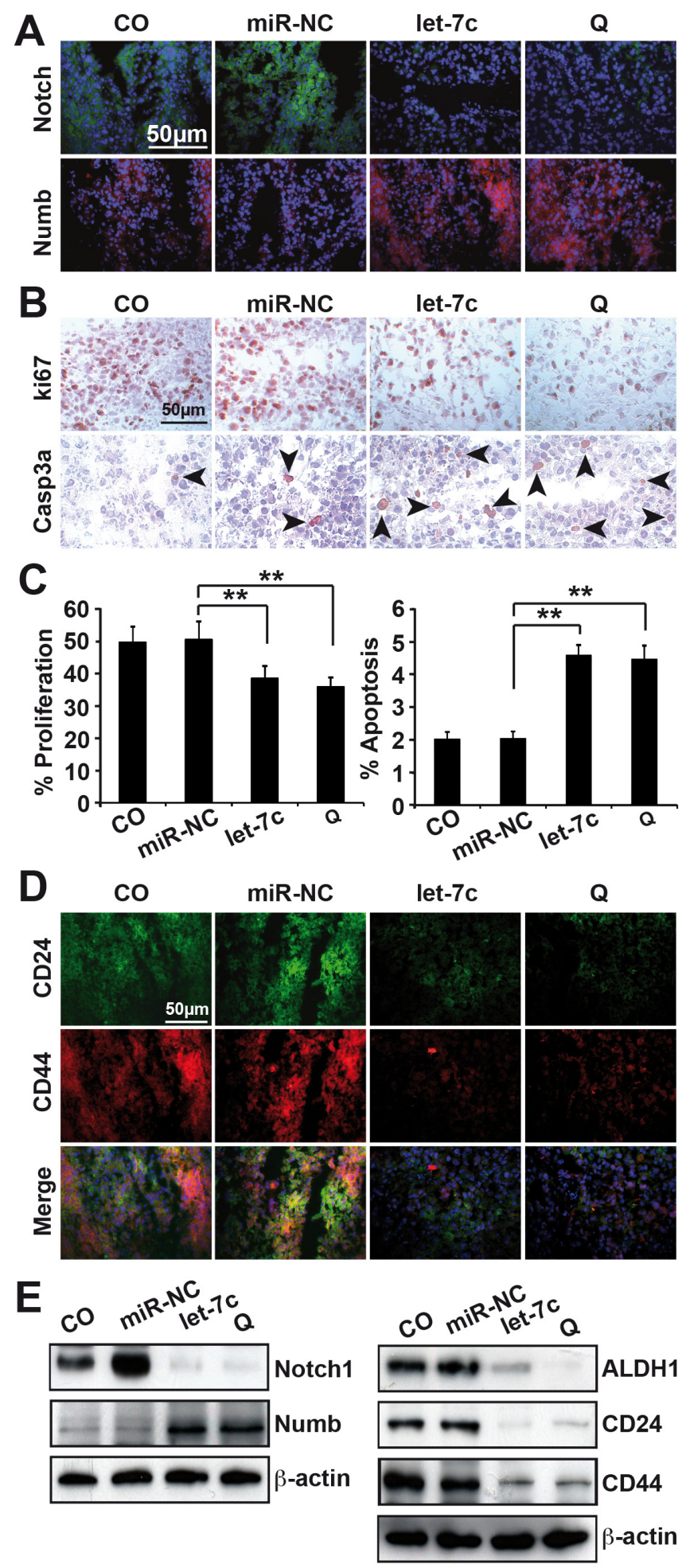

Figure 6: Immunofluorescence staining of tumor tissue confirms the former results. A. Immunofluorescence staining of frozen tumor section with Notch and Numbl. The bar indicates $50 \mu \mathrm{m} \mathrm{B.} \mathrm{Immunohistochemistry} \mathrm{staining} \mathrm{of} \mathrm{the} \mathrm{frozen} \mathrm{tissue} \mathrm{section} \mathrm{with}$ the human proliferation marker Ki67 and the apoptosis marker cleaved fragment of activated caspase-3. The positive cells appear red to dark red and are marked by the black arrows. The bar indicates $50 \mu \mathrm{m}$. C. The numbers of positive cells were quantified in ten vision fields. D. Immunofluorescence staining of frozen tumor section with CD24 and CD44. E. Western blot analyses were performed for Notch1, Numb and the cancer stem cell markers ALDH1, CD24 and CD44. $\beta$-actin served as controls for equal conditions. All assays were performed in triplicates, repeated at least three times. The bar indicates $50 \mu \mathrm{m}$. The means $\pm \mathrm{SD}$ are shown. ${ }^{*} \mathrm{P}<0.05, * * \mathrm{P}<0.01$. 
quercetin have been observed [18]. Clinical trials with quercetin show tolerance of quercetin doses ranging from $60 \mathrm{mg} / \mathrm{m} 2(1.5 \mathrm{mg} / \mathrm{kg})-1700 \mathrm{mg} / \mathrm{m}^{2}$ (42.5 mg/kg) [19]. However, the therapeutic active doses of quercetin are difficult to be realized by nutrition alone. For example, to achieve about $500 \mathrm{mg}$ of quercetin, this would amount to the consumption of a liter of red wine $(19 \mathrm{mg}), 1 \mathrm{~kg}$ apples (140 mg), $1 \mathrm{~kg}$ yellow onions (347 mg) or $1 \mathrm{~kg}$ broccoli (30 mg) [20].

Therefore, we studied the quercetin-induced modulation of miR signaling in an attempt to facilitate the delivery of quercetin-induced effects to patients. Our microarray analysis showed 105 miRs differentially expressed between quercetin-treated and non-treated cells. Top amongst them were miRs reported to decrease tumor metastasis and invasion (miR-194, miR-103, miR-29) [21, 22], inhibit cell proliferation (let-7 family, miR-215) [23], induce apoptosis (miR-125) [24], and tumor suppressors (let-7 family, miR-125, miR-106) $[25,26]$ to mention but a few.

Let-7 is a tumor suppressor miR and one of the earliest identified miRs per se. The expression of let7 family members is down-regulated in many types of cancer when compared to normal tissue and during tumor progression, it is associated with poor prognosis $[23,27]$. In this study, we show that the expression of let-7c is low in pancreatic cancer. While this is not new but confirms already published reports $[15,16]$, we show for the first time that quercetin treatment results in the transcriptional activation of let-7c by directly targeting the Numbl 3'-UTR. MiRs induce or repress gene expression via posttranscriptional regulatory mechanisms such as increased mRNA levels, re-localization of mRNAs/ miR-protein complexes (mRNPs), mRNP modification and alteration of the translation machinery [28]. Our data show that Numbl gene expression was induced by an increase of it's mRNA levels. Thus Numbl is upregulated and Notch signaling activities are antagonized. The molecular mechanism for this unique relationship depends most likely on the Notch1 receptor ubiquitination and degradation of the Notch1 intracellular domain (NCID), as described [29].

The Notch signaling pathway has been found to function as a down-regulator in osteoclastogenesis and osteoblastogenesis in in vitro and in vivo studies [30, 31]. Over-expression of Notch inhibited BMP-2-induced osteoblast differentiation, and Numbl was observed to support differentiation [32]. While our results are based on heterogeneous ALP activity, it is in line with these studies indicating that treatment with quercetin or let-7c leads to osteoblastic differentiation of PDA cells. The poor osteoblastic differentiation observed with PANC-1 is most likely due to its well-documented poor differentiation competence [33].

Therapeutic delivery of miR mimics known as miR replacement therapy is used to restore a loss of function.
Inhibition of tumor growth in human non-small cell lung cancer xenografts and the KRAS-G12D transgenic mouse model have resulted from therapeutic delivery of let-7 in either the form of a let-7 mimic or a virus [34]. This supports our data of tumor growth inhibition of PDA cells xenografts in the chick egg model by let-7c. miR-34 is the first miR replacement therapy in clinical trials by the company Mirna Therapeutics, with let-7 in the pipeline [35]. The miR mimics are delivered via a liposomal delivery technology called SMARTICLES $\AA$, which forms particles of $\sim 120 \mathrm{~nm}$ and are cationic at low $\mathrm{pH}$ and neutral or anionic at neutral or higher $\mathrm{pH}$.

Irrespective of the great and thrilling prospects of miRs, essential obstacles mostly involving it's delivery and cost-effective pre-clinical models remain to be scaled afore clinical applications. Till date, there is no single non-viral miRNA delivery model for in vivo studies that is cheap, rapid, reproducible and reliable. The mostly used animal models are mouse or rat with high administrative and ethical barriers. Hence, we propose the fertilized chick egg model as a robust alternative. Some of the arguments for the fertilized chick egg model include its naturally immunodeficiency and ability to receive transplantations from different tissues and species without species-specific restrictions, allowance of analyses of real time changes in morphology of cancer cells in its microcirculation, fast tumor growth between 2 and 5 days after transplantation, low cost, simplicity with little or no ethical and administrative hurdles [36]. Our results show concrete evidence that a significant percentage of the candidate $\mathrm{miR}$ is delivered to the tumor and the result could be correlated to data obtained from the in vitro analyses. Most importantly, it has a vast range of applications from proliferation studies to metastases, angiogenesis, toxicity etc.

In conclusion, our study demonstrates that let$7 \mathrm{c}$, which is down regulated in pancreatic cancer, is induced by quercetin. Numbl is activated by let-7c via posttranscriptional increase of its mRNA levels and Notch is antagonized (Supplementary Figure 2). The overall outcome is the inhibition of tumor growth, progression markers and increase in apoptosis.

\section{MATERIALS AND METHODS}

\section{Tumor cell lines}

AsPC-1, CRL-4023 and PANC-1 pancreatic cancer cell lines were obtained from the American Type Culture Collection (Manassas, VA, USA). The established cell lines were recently authenticated by a commercial service (Multiplexion, Heidelberg, Germany). The human, primary pancreatic cancer cell line ASANPaCa was kindly provided by Dr. Nathalia Giese and is described [37]. To maintain the authenticity of the cell lines, frozen stocks were prepared from initial stocks, and every three 
months a new frozen stock was used for experiments. Monthly testing ensured mycoplasma negative cultures. Cells were cultured in DMEM (PAA, Pasching, Austria) supplemented with 10\% heat-inactivated FCS (Sigma, Deisenhoffen, Germany) and $25 \mathrm{mmol} / \mathrm{L}$ HEPES (PAA, Pasching, Austria).

\section{Quercetin preparation}

Quercetin (2-(3,4-Dihydroxyphenyl)-3,5,7trihydroxy-4H-1-benzopyran-4-one) was purchased from Sigma-Aldrich (St. Louis, MO, USA). It was dissolved in cell culture grade Dimethyl Sulfoxide (DMSO) and added directly to the culture medium.

\section{Colony-forming assay}

The cells were harvested $24 \mathrm{~h}$ after lipofection or $12 \mathrm{~h}$ after treatment with quercetin (Sigma, Deisenhoffen, Germany). Then the cells were re-suspended in DMEM medium supplemented with $10 \%$ FCS and plated at a density of 500 cells/well in 6-well tissue culture plates. The cultures were maintained under standard culture conditions for fourteen days and the assay was performed as described [38].

\section{Wound healing assay}

The cells were harvested $24 \mathrm{~h}$ after lipofection or $12 \mathrm{~h}$ after treatment with quercetin (Sigma, Deisenhoffen, Germany). Then the cells were re-suspended in DMEM medium supplemented with $10 \%$ FCS and plated at $5 \mathrm{x}$ $10^{5}$ cells $/ \mathrm{ml}$ in Culture-Inserts ready to use in a $\mu$-Dish (ibiTreat, item \#: 80241, IBIDI, Martinsried, Germany), which allows high-resolution microscopy in a 35-mm Petri dish with $12-\mathrm{mm}$ walls. At about $100 \%$ cell confluence, the inserts were taken out to leave a $500-\mu \mathrm{M}$ gap in each dish and fresh media was added. Twenty-four hours and 48 $\mathrm{h}$ later, images were taken with a Nikon Eclipse TS 100-F inverted microscope equipped with a camera. Five images per treatment were collected. Analyses of the images were performed using ibidi's WimScratch Quantitative Wound Healing Image Analysis (item \#: 30002, IBIDI).

\section{Evaluation of osteoblastic differentiation}

Cells were seeded at a density of $4.5 \times 10^{4}$ cells/well in 6 -well cell culture plates. At $70-90 \%$ cell confluence, cells were treated with $50 \mu \mathrm{M}$ quercetin for $96 \mathrm{~h}$, or were transfected with mirVana ${ }^{\mathrm{TM}}$ mimics of hsa-let-7c or a noncoding miR control (miR-NC) for $96 \mathrm{~h}$ (Thermo Fischer Scientific, Waltham, MA USA) using lipofectamine RNAiMax reagent (Life Technologies, Darmstadt, Germany), or were left untransfected. The cells were washed, fixed with methanol, washed and incubated with FAST BCIP/NBT (Sigma-Aldrich, St. Louis, Missouri, USA), washed, and the deep bluish purple color of alkaline phosphatase expressing osteoblasts was visualized under $10 \times$ magnification using an inverted microscope (Nikon Eclipse TS100; Nikon GmbH, Düsseldorf, Germany). The Sigma FAST BCIP/NBT active substrate solution containing BCIP $(0.15 \mathrm{mg} / \mathrm{ml})$, NBT $(0.30 \mathrm{mg} / \mathrm{ml})$, Tris buffer (100 mM), and $\mathrm{MgCl} 2(5 \mathrm{mM}), \mathrm{pH} 9.25-9.75$ was prepared by dissolving one tablet in $10 \mathrm{ml}$ of water.

\section{Western blot analysis}

Western blot analysis was performed as previously described [38]. Antibodies used include: rabbit antibodies against human Notch (Cell Signaling Technology, Danvers, USA), Numbl (Genetex, Irvine, USA), CD44, ALDH1 (both from Abcam, Cambridge, UK) and mouse antibodies against CD24 (Abcam).

\section{Immunohistochemistry and immunofluorescence staining}

Stainings were performed on $6-\mu \mathrm{m}$ frozen tissue sections or in established cell lines growing in chambers of the Nunc ${ }^{\circledR}$ Lab-Tek chamber Slide ${ }^{\mathrm{TM}}$ system (SigmaAldrich, St. Louis, Missouri, USA) as previously described $[38,39]$.

\section{MicroRNA transfection}

$50 \mathrm{nM}$ miR let-7c mimic or non-coding miR (Ambion-Thermo Fisher Scientific Inc., Dreieich, Germany) were transfected into the cells using Lipofectamine 2000 (Invitrogen-Thermo Fisher Scientific Inc., Dreieich, Germany) or Lipofectamine RNAiMAX (Invitrogen) transfection reagent using the reverse transfection method as described in the manufacturer's instructions.

\section{Dual-Luciferase ${ }^{\circledR}$ reporter assay}

Cells were seeded at a density of $1 \times 10^{4}$ cells per well of a 96-well plate and co-transfected with $50 \mathrm{nM}$ miR mimics, $25 \mathrm{ng} /$ well of firefly luciferase plasmid and $50 \mathrm{ng} /$ well renilla luciferase reporter construct expressing Numbl 3'-UTR (BioCat, Heidelberg, Germany). The cells were lysed in Passive Lysis Buffer of the Dual Luciferase ${ }^{\circledR}$ Assay System (Promega, Mannheim, Germany) at $48 \mathrm{~h}$ post-transfection. Renilla and firefly luciferase activities were measured on the FLUOstar OPTIMA instrument (BMG Labtech, Ortenberg, Germany).

\section{MiR microarray profiling and analysis}

The Agilent Human miRNA Microarray (Release 19.0) was used for microRNA profiling. The crude array data were analyzed using linear regression model for microarrays (LIMMA). The Benjamini and Hochberg $(\mathrm{BH})$ algorithm was used to correct for multiple testing 
and False Discovery Rate (FDR). Gene ontology analysis confirmed the association of the identified set of miRNA candidates with specific biological processes and molecular functions. The microarray data was uploaded to Array Express with the accession number E-MTAB-4718.

\section{MiR in silico analysis}

The miR target predictions based on miRanda, TargetScan and PicTar were downloaded from http:// www.microrna.org [40], http://www.targetscan.org [41] and from http://www.pictar.org [42], respectively. "Good" mirSVR score refers to miR targets with $<-0.1$ score, and "non-good" mirSVR score refers to targets with $>$ -0.1 score obtained from the support vector regression algorithm mirSVR, available with miRanda predictions from http://www.microrna.org.

\section{Taqman miR real-time qPCR}

cDNA was synthesized from 500 ng total RNA using the Taqman ${ }^{\circledR}$ Reverse Transcription Reagents (Thermo Fisher Scientific, Dreieich, Germany) according to the manufacturers' instructions. Quantitative levels of hsa-let7c (5'-uga ggu agu agg uug uau ggu u-3') were measured from $1 \mu \mathrm{l}$ of synthesized cDNA using their respective primer assays and RNU44 as endogenous control (Applied Biosystems, Darmstadt, Germany). Notch1 and Numbl mRNA levels were quantified from $2 \mu \mathrm{l}$ of synthesized cDNA using the TaqMan Gene Expression Assay and primers for Notch1, Numbl and GAPDH (Thermo Fisher Scientific). The PCR was run on the StepOne Real-Time PCR System (Applied Biosystems). Changes in the relative concentration were calculated with the second derivative maximum method $2^{-\Delta \mathrm{CT}}[43]$. $\Delta \mathrm{CT}$ was calculated by subtracting the CT of the housekeeping gene from the CT of the gene of interest. The fold change was generated using the equation $2^{-\Delta \Delta \mathrm{CT}}$.

\section{Tumor xenotransplantation to fertilized chick eggs and in ovo treatment}

ASANPaCa cells were left untransfected, treated with $50 \mu \mathrm{M}$ quercetin or lipofected with $50 \mathrm{nM}$ mirVana $^{\mathrm{TM}}$ mimics of hsa-let-7c or a non-coding miR (Thermo Fischer Scientific, Waltham, MA USA) using lipofectamine RNAiMax reagent (Life Technologies, Darmstadt, Germany). Forty-eight hours later, the cells were transplanted to the CAM of fertilized chick eggs, 50 eggs per group, at day 9 of embryonal development. The fertilized eggs were from highly genetically identical hybrid Lohman Brown (LB) chicks and were delivered by a local ecological hatchery (Geflügelzucht Hockenberger, Eppingen, Germany). Before, the eggs were prepared for transplantation by exposure of the CAM at day 4 of development as we described recently [44]. Five days post- transplantation at day 14, control eggs were injected with $50 \mu \mathrm{l}$ chick saline $\left(7.2 \mathrm{~g} \mathrm{NaCl}, 0.37 \mathrm{~g} \mathrm{KCl}, 0.23 \mathrm{~g} \mathrm{CaCl}_{2}\right.$ per Liter $\mathrm{ddH}_{2} \mathrm{O}, \mathrm{pH}$ 7.2-7.3) [45], and eggs harboring miR-pretreated xenografts with $50 \mu \mathrm{l}$ miR-lipofectamine mix containing $50 \mathrm{nM}$ has-let-7c mimics or in chick saline using an Omnican ${ }^{\circledR} \mathrm{F}$ fine dosage syringe with an integrated $30 \mathrm{G} \times 8 \mathrm{~mm}$ needle $(\mathrm{B}$. Braun Melsungen AG, Melsungen, Germany). The xenograft tumors were resected 4 days later at day 18 , after humane euthanasia of the chick embryo [46]. All embryos that died before day 17 were excluded from further analyses. The tumor volumes were estimated using the following formula: volume $=4 / 3 \times \prod \times \mathrm{r}^{3}(\mathrm{r}=1 / 2 \times \sqrt{ }$ of diameter $1 \times$ diameter 2$)$ [47].

\section{Statistical analysis}

The quantitative data are presented as the mean $\pm \mathrm{SD}$. The data were analyzed using Student's $t$-test for statistical significance. $P<0.05$ was deemed to be statistically significant.

\section{ACKNOWLEDGMENTS}

We thank the microarray unit of the Genomics and Proteomics Core Facility of the German Cancer Research Center (DKFZ) Heidelberg for providing the Illumina Whole-Genome Expression Bead chips and related services. We are also grateful to Dr. W. Gross for providing Histo 3.0 customized image analysis software, and Jutta Mohr and Sebastian Faus for excellent technical assistance.

\section{CONFLICTS OF INTEREST}

None of the authors has a conflicts of interest to disclose regarding the publication of the present manuscript.

\section{Author contributions}

$\mathrm{IH}$ : Concept and design

CN, NB, ZZ, AA: Development of methodology

$\mathrm{CN}, \mathrm{JG}$ : Acquisition of data

$\mathrm{CN}, \mathrm{AB}$ : Analysis and interpretation of data

$\mathrm{CN}$, IH: Writing, review and/or revision of the manuscript.

\section{REFERENCES}

1. Siegel R, Ma J, Zou Z, Jemal A. Cancer statistics, 2014. CA Cancer J Clin. 2014; 64:9-29.

2. Ryan DP, Hong TS, Bardeesy N. Pancreatic adenocarcinoma. N Engl J Med. 2014; 371:2140-2141. 
3. Cioffi M, Trabulo SM, Sanchez-Ripoll Y, Miranda-Lorenzo I, Lonardo E, Dorado J, Reis Vieira C, Ramirez JC, Hidalgo M, Aicher A, Hahn S, Sainz B, Jr. and Heeschen C. The miR-17-92 cluster counteracts quiescence and chemoresistance in a distinct subpopulation of pancreatic cancer stem cells. Gut. 2015; 64:1936-1948.

4. Neesse A, Gress TM. Emerging role of microRNAs to tackle drug resistance in pancreatic cancer. Gut. 2015; 64:1842-1843.

5. Yates LA, Norbury CJ, Gilbert RJ. The long and short of microRNA. Cell. 2013; 153:516-519.

6. Lin S, Gregory RI. MicroRNA biogenesis pathways in cancer. Nat Rev Cancer. 2015; 15:321-333.

7. Reinhart BJ, Slack FJ, Basson M, Pasquinelli AE, Bettinger JC, Rougvie AE, Horvitz HR, Ruvkun G. The 21-nucleotide let-7 RNA regulates developmental timing in Caenorhabditis elegans. Nature. 2000; 403:901-906.

8. Patterson M, Gaeta X, Loo K, Edwards M, Smale S, Cinkornpumin J, Xie Y, Listgarten J, Azghadi S, Douglass SM, Pellegrini M, Lowry WE. let-7 miRNAs can act through notch to regulate human gliogenesis. Stem Cell Rep. 2014; 3:758-773.

9. Miele L, Miao H, Nickoloff BJ. NOTCH signaling as a novel cancer therapeutic target. Curr Cancer Drug Targets. 2006; 6:313-323.

10. Kopan R, Ilagan MXG. The Canonical Notch Signaling Pathway: Unfolding the Activation Mechanism. Cell. 2009; 137:216-233.

11. Flores AN, McDermott N, Meunier A, Marignol L. NUMB inhibition of NOTCH signalling as a therapeutic target in prostate cancer. Nature Rev Urol. 2014; 11:499-507.

12. Appari M, Babu KR, Kaczorowski A, Gross W, Herr I. Sulforaphane, quercetin and catechins complement each other in elimination of advanced pancreatic cancer by miRlet-7 induction and K-ras inhibition. Int J Oncol. 2014; 45:1391-1400.

13. Nam JS, Sharma AR, Nguyen LT, Chakraborty C, Sharma G, Lee SS. Application of Bioactive Quercetin in Oncotherapy: From Nutrition to Nanomedicine. Molecules. 2016; 21:108.

14. Kawahara T, Kawaguchi-Ihara N, Okuhashi Y, Itoh M, Nara N, Tohda S. Cyclopamine and quercetin suppress the growth of leukemia and lymphoma cells. Anticancer Res. 2009; 29:4629-4632.

15. Patel K, Kollory A, Takashima A, Sarkar S, Faller DV, Ghosh SK. MicroRNA let-7 downregulates STAT3 phosphorylation cancer cells by increasing SOCS3 expression. Cancer Lett. 2014; 347:54-64.

16. Jiao LR, Frampton AE, Jacob J, Pellegrino L, Krell J, Giamas G, Tsim N, Vlavianos P, Cohen P, Ahmad R, Keller A, Habib NA, Stebbing J and Castellano L. MicroRNAs Targeting Oncogenes Are Down-Regulated in Pancreatic Malignant Transformation from Benign Tumors. Plos One. $2012 ; 7$.
17. Nothlings U, Murphy SP, Wilkens LR, Boeing H, Schulze MB, Bueno-De-Mesquita HB, Michaud DS, Roddam A, Rohrmann S, Tjonneland A, Clavel-Chapelon F, Trichopoulou A, Sieri S, Rodriguez L, Ye WM, Jenab M, et al. A food pattern that is predictive of flavonol intake and risk of pancreatic cancer. Am J Clin Nutr. 2008; 88:1653-1662.

18. Mulholland PJ, Ferry DR, Anderson D, Hussain SA, Young AM, Cook JE, Hodgkin E, Seymour LW, Kerr DJ. Preclinical and clinical study of QC12, a water-soluble, prodrug of quercetin. Ann Oncol. 2001; 12:245-248.

19. Ferry DR, Smith A, Malkhandi J, Fyfe DW, deTakats PG, Anderson D, Baker J, Kerr DJ. Phase I clinical trial of the flavonoid quercetin: Pharmacokinetics and evidence for in vivo tyrosine kinase inhibition. Clin Cancer Res. 1996; 2:659-668.

20. Zhou W, Kallifatidis G, Baumann B, Rausch V, Mattern J, Gladkich J, Giese N, Moldenhauer G, Wirth T, Buchler MW, Salnikov AV, Herr I. Dietary polyphenol quercetin targets pancreatic cancer stem cells. Int J Oncol. 2010; 37:551-561.

21. Gebeshuber CA, Zatloukal K, Martinez J. miR-29a suppresses tristetraprolin, which is a regulator of epithelial polarity and metastasis. Embo Rep. 2009; 10:400-405.

22. Martello G, Rosato A, Ferrari F, Manfrin A, Cordenonsi M, Dupont S, Enzo E, Guzzardo V, Rondina M, Spruce T, Parenti AR, Daidone MG, Bicciato S, Piccolo S. A MicroRNA Targeting Dicer for Metastasis Control. Cell. 2010; 141:1195-U1176.

23. Johnson CD, Esquela-Kerscher A, Stefani G, Byrom N, Kelnar K, Ovcharenko D, Wilson M, Wang XW, Shelton J, Shingara J, Chin L, Brown D, Slack FJ. The let-7 MicroRNA represses cell proliferation pathways in human cells. Cancer Res. 2007; 67:7713-7722.

24. Gong J, Zhang JP, Li B, Zeng C, You K, Chen MX, Yuan Y, Zhuang SM. MicroRNA-125b promotes apoptosis by regulating the expression of Mcl-1, Bcl-w and IL-6R. Oncogene. 2013; 32:3071-3079.

25. Nohata N, Hanazawa T, Kinoshita T, Okamoto Y, Seki N. MicroRNAs function as tumor suppressors or oncogenes: Aberrant expression of microRNAs in head and neck squamous cell carcinoma. Auris Nasus Larynx. 2013; 40:143-149.

26. Shohet J, Ludwig A, Chen ZW, Ghosh R, Sikorski D, Patterson D, Coafra C, Gunaratne P. High Throughput Sequencing and Chromatin Immunoprecipitation (ChipSeq) Identifies Mycn-Regulated Micrornas as Tumor Suppressors and Oncogenes in Neuroblastoma. Ped Blood \& Cancer. 2010; 54:840-841.

27. Thomson JM, Newman M, Parker JS, Morin-Kensicki EM, Wright T, Hammond SM. Extensive post-transcriptional regulation of microRNAs and its implications for cancer. Genes \& Dev. 2006; 20:2202-2207. 
28. Vasudevan S, Tong YC, Steitz JA. Switching from repression to activation: MicroRNAs can up-regulate translation. Science. 2007; 318:1931-1934.

29. McGill MA, McGlade CJ. Mammalian numb proteins promote notch1 receptor ubiquitination and degradation of the notch1 intracellular domain. J Biol Chem. 2003; 278:23196-23203.

30. Deregowski V, Gazzerro E, Priest L, Rydziel S, Canalis E. Notch 1 overexpression inhibits osteoblastogenesis by suppressing Wnt/beta-catenin but not bone morphogenetic protein signaling. J Biol Chem. 2006; 281:6203-6210.

31. Ann EJ, Kim HY, Choi YH, Kim MY, Mo JS, Jung J, Yoon JH, Kim SM, Moon JS, Seo MS, Hong JA, Jang WG, Shore P, Komori T, Koh JT, Park HS. Inhibition of Notch1 Signaling by Runx2 During Osteoblast Differentiation. J Bone Min Res. 2011; 26:317-330.

32. Givogri MI, Schonmann V, Cole R, de Vellis J, Bongarzone ER. Notch1 and Numb genes are inversely expressed as oligodendrocytes differentiate. Dev Neurosc. 2003; 25:50-64.

33. Lieber M, Mazzetta J, Nelsonrees W, Kaplan M, Todaro G. Establishment of a Continuous Tumor-Cell Line (Panc1) from a Human Carcinoma of Exocrine Pancreas. Int $\mathbf{J}$ Cancer. 1975; 15:741-747.

34. Trang P, Medina PP, Wiggins JF, Ruffino L, Kelnar K, Omotola M, Homer R, Brown D, Bader AG, Weidhaas JB, Slack FJ. Regression of murine lung tumors by the let-7 microRNA. Oncogene. 2010; 29:1580-1587.

35. Beg MS, Borad M, Sachdev J, Hong DS, Smith S, Bader A, Stoudemire J, Kim S, Brenner A. Multicenter phase I study of MRX34, a first-in-class microRNA miR-34 mimic liposomal injection. Cancer Res. 2014; 74.

36. Ribatti D. The chick embryo chorioallantoic membrane as a model for tumor biology. Exp Cell Res. 2014; 328:314-324.

37. Heller A, Angelova AL, Bauer S, Grekova SP, Aprahamian M, Raykov Z, Rommeleare J, Volkmar M, Janssen JW-G, Herr I, Bauer N, Giese T, Gaida MM, Bergmann F, Hackert $\mathrm{T}$, Fritz S, et al. Establishment and characterization of a novel cell line, ASAN-PaCa, derived from human adenocarcinoma arising in intraductal papillary mucinous neoplasm of the pancreas. Pancreas. 2016; in press.
38. Kallifatidis G, Rausch V, Baumann B, Apel A, Beckermann BM, Groth A, Mattern J, Li Z, Kolb A, Moldenhauer G, Altevogt P, Wirth T, Werner J, Schemmer P, Buchler MW, Salnikov AV, et al. Sulforaphane targets pancreatic tumourinitiating cells by NF-kappa B-induced antiapoptotic signalling. Gut. 2009; 58:949-963.

39. Liu L, Salnikov AV, Bauer N, Aleksandrowicz E, Labsch S, Nwaeburu C, Mattern J, Gladkich J, Schemmer P, Werner J, Herr I. Triptolide reverses hypoxia- induced epithelialmesenchymal transition and stem- like features in pancreatic cancer by NF- jB downregulation. Int J Cancer. 2014; 134:2489-2503.

40. Betel D, Wilson M, Gabow A, Marks DS, Sander C. The microRNA.org resource: targets and expression. Nucleic Acids Res. 2008; 36:D149-D153.

41. Lewis BP, Burge CB, Bartel DP. Conserved seed pairing, often flanked by adenosines, indicates that thousands of human genes are microRNA targets. Cell. 2005; 120:15-20.

42. Krek A, Grun D, Poy MN, Wolf R, Rosenberg L, Epstein EJ, MacMenamin P, da Piedade I, Gunsalus KC, Stoffel M, Rajewsky N. Combinatorial microRNA target predictions. Nature Genetics. 2005; 37:495-500.

43. Livak KJ, Schmittgen TD. Analysis of relative gene expression data using real-time quantitative PCR and the 2(T) (-Delta Delta C) method. Methods. 2001; 25:402-408.

44. Liu L. SAV, Bauer N., Aleksandrowicz E., Labsch S., Nwaeburu C., Mattern J., Gladkich J., Schemmer P., Werner J., Herr I. Triptolide reverses hypoxia-induced epithelialmesenchymal transition and stem-like features in pancreatic cancer by NF-kB downregulation. Int J Cancer. 2013.

45. McCain ER, McLaughlin JS. (1999). Developmental and physiological aspects of the chicken embryonic heart. In: Karcher SJ, ed. Tested studies for laboratory teaching, pp. 85-100.

46. Aleksandrowicz E, Herr I. Ethical euthanasia and short-term anesthesia of the chick embryo. ALTEX. 2015; 32:143-147.

47. Balke M, Neumann A, Szuhai K, Agelopoulos K, August C, Gosheger G, Hogendoorn PC, Athanasou N, Buerger $\mathrm{H}$, Hagedorn M. A short-term in vivo model for giant cell tumor of bone. BMC Cancer. 2011; 11:241. 